\title{
Ectopic Gall Bladder Associated With Segmental Hypoplasia of Right Lobe of Liver: Two Cases
}

\author{
Authors \\ Keshaba Panigrahy ${ }^{1}$, Subhasish Panda ${ }^{2}$, Braja B Panda ${ }^{3}$, Savitri Bhagat ${ }^{4}$, \\ Shamim Nisa ${ }^{5}$, Bararuchi Dash ${ }^{6}$ \\ ${ }^{1,2}$ Resident, P.G., ${ }^{3,5,6}$ Associate Professor, P.G., ${ }^{4}$ Professor and Head, P.G.
}

Dept of Radiodiagnosis, V.S.S. Institute of Medical Sciences and Research, Burla, Sambalpur, Odisha, India Corresponding Author

Keshaba Panigrahy

Email:drkeshab2010@gmail.com

\begin{abstract}
The gallbladder is a pear shaped organ lying in the inferior margin of liver, between the right and left lobes. Its primary function is to store and concentrate bile produced by the liver. The gallbladder is affected by a large number of congenital anomalies which may affect its location, number, size and form. Some of these malformations are very rare and may lead to misdiagnosis. An ectopic gallbladder is a rare condition and can be misdiagnosed as agenesis of the organ or as a cystic mass when intrahepatic. We present two cases of ectopic gallbladder and in both cases it was associated with partial agenesis of right lobe of liver.
\end{abstract}

\section{Introduction}

The gallbladder is an abdominal organ and adequately examined by ultrasonography. Whenever any abnormality is detected or gallbladder is not visualised in its normal position, we have to do CT for proper diagnosis.

We present two cases: in the 1 st case the gallbladder could not be visualised by ultrasonography and in the 2nd case the gallbladder was visualised in an ectopic location. We completed the imaging approach of the patients by providing a Computed Tomography image. In both cases there was associated partial agenesis of right lobe of liver.

\section{Case Report}

Case 1

A 50 year old female was referred to our department with recurrent epigastric pain. On ultrasound, her gall bladder couldn't be visualised at the gall bladder fossa. Instead, gas filled bowel loops were noted in the region of right hypochondrium obscuring the liver whose right lobe was hypoplastic (Fig 1) Retrospectively after CT Scan, the ectopic gall bladder was located.

Contrast Enhanced Computed Tomography was carried out thereafter, which revealed an ectopic location of the gall bladder related to the anterolateral surface of the liver. (Fig 3)

Examination of the gall bladder demonstrated wall thickening with multiple internal radiodense calculi. 


\section{JMSCR Vol||05||Issue||02||Pages 17343-17345||February}

Scanty liver parenchyma was situated between the falciform ligament and the gall bladder suggestive of a small segment V and hypoplastic segment VIII. Hepatic flexure of the colon along with pylorus of the stomach were displaced supero-laterally with respect to the right lobe of the liver.(Fig 4)

The Spleen was enlarged (span of $23 \mathrm{~cm}$ ) with normal homogenous contrast enhancement. Multiple Dilated tortuous contrast filled vascular channels were seen at the splenic hilum.(Fig 2)

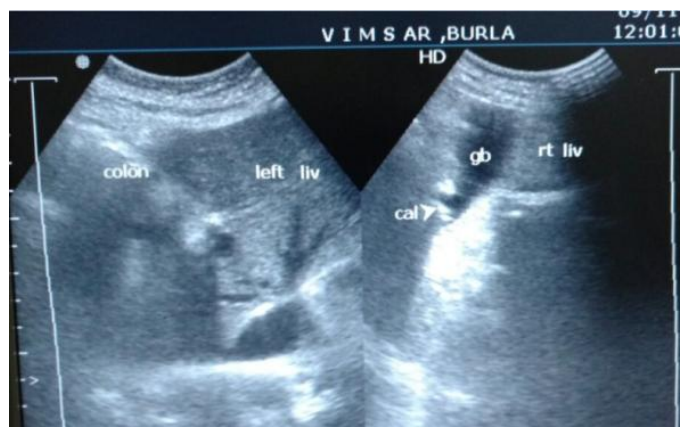

Fig 1. USG Abdomen showing hypoplasia of right lobe of liver and an echogenic calculus in the ectopic gall bladder.

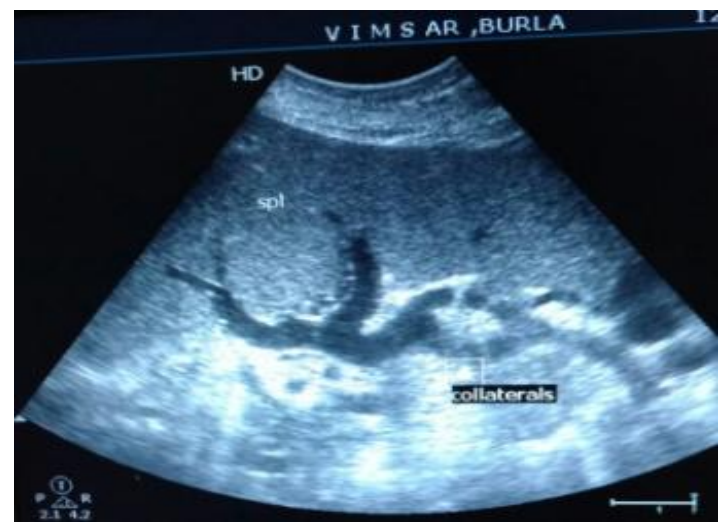

Fig 2. USG abdomen showing dilated splenorenal collaterals

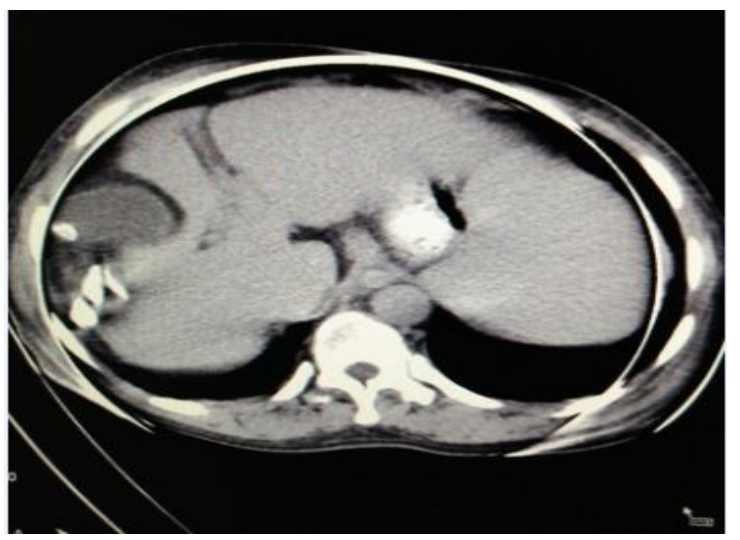

Fig 3. Axial CT Scan of the abdomen showing suprahepatic gall bladder with cholelithiasis

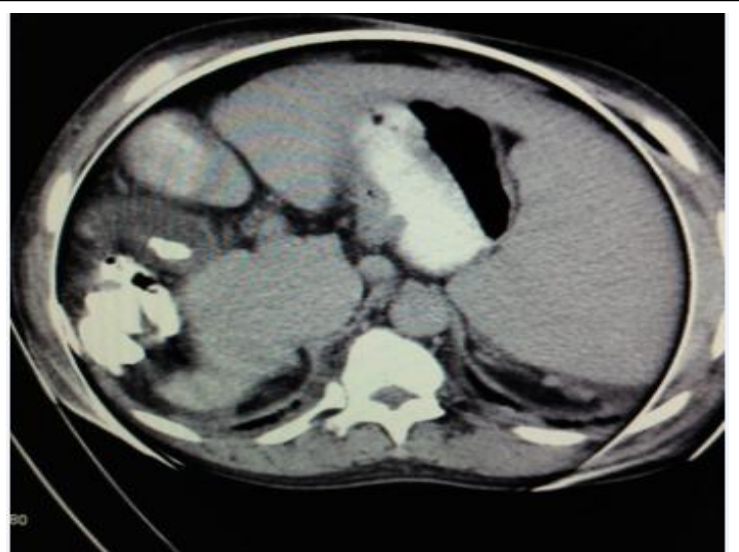

Fig 4. Axial CT Scan few sections caudad showing displacement of pylorus of stomach and the contrast filled hepatic flexure.

\section{Case 2}

A 65 year old male underwent CT Scan in our department for evaluation of umbilical hernia. Axial sections demonstrated diffuse linear air densities along his intrahepatic biliary radicles consistent with Pneumobilia. Incidentally, his gall bladder was localised laterally in close proximity to the anterior abdominal wall. Aplasia of segment $\mathrm{V}$ of the liver was associated (Fig 5)

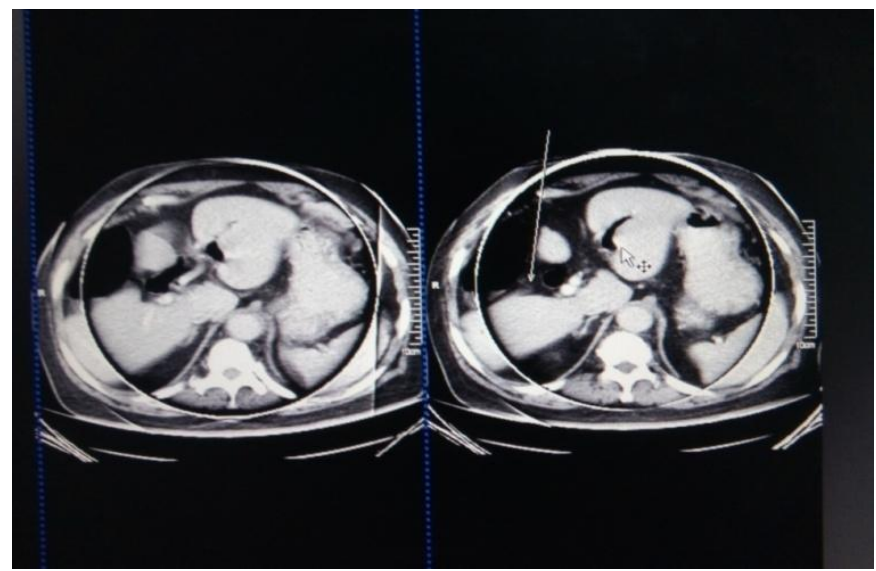

Fig 5. Axial Computerised Tomography depicts the ectopic gall bladder located anterior to hepatic segment VI which has a luminal air- fluid level suggestive of sludge. The Long arrow denotes the aplastic segment $\mathrm{V}$ of Liver. Short arrow demarcates associated pneumobilia.

\section{Discussion}

The gall bladder derives as an out pouching from the embryonic billiary tree which appears in the ventral wall of primitive midgut during the 4 th week 
of gestation. The proximal portion of the pouch forms the cystic duct and distal portion form the gallbladder. During initial development the gallbladder lies in the intrahepatic position and subsequently it migrates to the surface of the liver and acquires a peritoneal covering. Failure to migrate may lead to an intrahepatic gallbladder.

The normal position of the gallbladder is under the right lobe of liver in the interlobar fissure between the right lobe and medial segment of the left lobe. An ectopic gallbladder is a rare entity ,the incidence being $0.1-0.7 \%(0)$. The ectopic locations may be intrahepatic (most common), suprahepatic, suprarenal, with in the abdominal wall or rarely in the falciform ligament. The diagnosis of an ectopic gall bladder should be suspected whenever the gallbladder cannot be localised in its usual location $(4,5)$ by ultrasonography. The gallbladder's ectopic position can be identified by $\mathrm{CT}$ and angiography by identifying the cystic artery ${ }^{(6)}$. The ectopic position causes impaired function and leads to stasis predisposing the patient to cholelithiasis and cholecystitis (seen in Case 1). Sometimes the typical symptoms and signs of acute cholecystitis may be absent if the gallbladder is situated away from the peritoneum. So cholecystectomy should be seriously considered for ectopic gall bladder, even in asymptomatic cases.

It is a major source of clinical confusion as was the case in our patients where a right lateral location was encountered ${ }^{(3)}$. Association of this rare entity with hypoplasia or aplasia of the right lobe of liver has been reported in the past ${ }^{(1,2)}$ and is unusual. All these facts indicate the rarity of our cases. Peristaltic movements of the displaced stomach, duodenum, and the transverse colon can initiate torsion. Proper diagnosis of this rare phenomenon can avoid a surgical mishaps. Enlarged spleen along with vascular channels in case 1 was indicative of portal hypertension and portosystemic anastomoses which was thought to be secondary to periportal fibrosis.

Pneumobilia in Case 2 was thought to be secondary to hypotonic Sphincter of Oddi which is a common phenomenon in older patients.

\section{Conclusion}

The possibility of an ectopic gallbladder should always be considered and Contrast Enhanced Computerised Tomograpy advised whenever there is a failure to localize it in its normal anatomical position on routine imaging. Once such a condition is diagnosed, Cholecystectomy is imperative. The liver parenchyma must be examined in these patients to rule out associated hypoplasia of any of its segments.

\section{References}

1. Rather AT, Khan HS, Singh M, Choh AN. A Rare Case of Suprahepatic Gall bladder with Phocomelia and Pancytopenia: Detected by Tc-99m Mebrofenin Scintigraphy. World Journal of Nuclear Medicine 2013; 12:41-44.

2. Youngworth LD, Peters JC, Perry MC. The suprahepatic gallbladder. Radiology 1983;149:57-8.

3. Faintuch J, Machado MC, Raia AA. Suprahepatic gallbladder with hypoplasia of the right lobe of the liver. Arch Surg 1980;115:658-9

4. Bockman DE, Freeny PC: Anatomy anomalies of the biliary tree. Laparos Surg 1992; 1: 92.

5. Meilstrup JW, Hopper KD, Thieme GA. Imaging of gallbladder variants. American Journal of Roentgenology 1991; 157 no. $6,: 1205-1208$

6. Hammond Ian. Unusual causes of sonographic nonvisualization or nonrecognition of the gallbladder: A review. Journal of Clinical Ultrasound 1988;16:2, 77-85

7. M. A. Carbajo, J.C. Martin del Omo, J. I. Blanco et al., "Congenital malformations of the gallbladder and cystic duct diagnosed by laparoscopy: High surgical risk,' Journal of the society of laparoendoscopic surgeons, vol 3, no. 4, pp. 319-321, 1999. 